\title{
NetMoAd: Scalability, Dependability, and Flexibility in Ad hoc Networks
}

\author{
Gísli Hjálmtýsson \\ Network Systems and Services Laboratory \\ Reykjavík University, Reykjavík, Iceland \\ gisli@ru.is
}

\begin{abstract}
Wireless ad-hoc networking is increasingly seen as critical part of ubiquitous self-configuring networking environments, including home and sensory networks and networks required to support ideas of pervasive computing. While basic principles of networking remain intact the large number of networked elements many of which exhibit mobility while being limited in power and other aspects introduces challenges not seen in traditional networks. The complexity of these future networks is further aggravated as increasing flora of applications exploit increasing diversity in of network and user equipment. Ad-hoc networking requires these devices to play a dual role of an end-system acting in its own interests and also the role of a router forwarding package on behalf of other devices. We foresee a wide range of operating environments from highly volatile - with rapidly moving mobiles and fluttering availability, to semi stable sensory environments - where node failures or external changes are the primary sources of network volatility.
\end{abstract}

\section{Framework}

We have recently started a project on ad-hoc networking focused on scalability and dependability and flexibility by targeting three main areas: reachability and routing, aggregation of routing information and on nodal architecture and operating system support for the duality required for the networked elements. While based on our own prior work and that of others, the project is in its early stages with limited amount of results so far. This abstract briefly describes the project, our approaches and our preliminary results.

The research is conducted at the Network Systems and Services Laboratory at Reykjavík University, lead by Dr. Gísli Hjálmtýsson, professor of Computer Science. The effort constitutes a new direction for our research on network and router architectures, and novel network systems and services. In particular, we aim to build on our prior and ongoing work on active and programmable networking $[1,2]$ and self-configuring services [3,4], rapid restoration [5,6,7], and service dependability [8]. In addition to Professor Hjálmtýsson, Prof. Heimir Pór Sverrisson and two Ph.D. students are involved at this early stage of the project.

The project is currently supported by a grant from the Icelandic Centre for Research, with the aim of applying for full funding to the European Commission project. Currently this project is done by Reykjavík University, but part of our current 
funding is attributed to building a collaboration group and to connect our efforts with related work performed elsewhere, particularly for the purposes of joining forces to seek EU funding.

\section{Objective}

Immediate objective of the project is to evolve to the level of maturity and group size (collaboration) to become viable project to be funded by the European Commission. Technical project objectives are to achieve scalability in routing, to achieve dependability in highly mobile ad-hoc environments, and to understand the role of nodal architecture in these two.

\section{Challenges}

We foresee as a primary challenge to achieve aggregation in network information, particularly routing/forwarding information. Also, whereas reliability is well defined in traditional transport networks, finding ways to make connectivity and availability of network services dependable over a volatile ad-hoc network appears to be a significant challenge.

\section{Task Performed to Tackle Challenges}

Initially, we will perform a significant amount of analysis and simulation to explore the range of potential options and deepen our understanding of the underlying problem principles. At the same time, we will implement and experiment with relevant node OS support to rationalize the practicality of these potential options. Our goal is that the result of this initial result will be concrete protocol and mechanism proposals, validated with analytical models, simulation and implementation on network elements; moreover, that the implementation and experimentation will result a set of requirements for system support.

\section{Project Organization}

Wireless ad-hoc networking is increasingly seen as critical part of ubiquitous selfconfiguring networking environments, including home and sensory networks and networks required to support ideas of pervasive computing. While basic principles of networking remain intact the large number of networked elements many of which exhibit mobility while being limited in power and other aspects introduces challenges not seen in traditional networks. The complexity of these future networks is further aggravated as increasing flora of applications exploit increasing diversity in of network and user equipment. Ad-hoc networking requires these devices to play a dual 
role of an end-system acting in its own interests and also the role of a router forwarding package on behalf of other devices. We foresee a wide range of operating environments from highly volatile - with rapidly moving mobiles and fluttering availability, to semi stable sensory environments - where node failures or external changes are the primary sources of network volatility.

\subsection{Scalability of Reachability and Routing}

The first focal point of our effort is reachability and routing. While in a (semi-) stable environments automatic allocation of addresses may be optimized to aggregate routing, in more volatile settings address allocation is reduced to ensuring uniqueness thus effectively eliminating route aggregation. In small static environments it is viable for each node to keep a routing entry for every host. This is in fact common in modern layer-2 devices that self-configure a MAC layer forwarding table by observing the transiting traffic. However, this approach is infeasible either in highly volatile environments or in networks having a large number of elements. We expect that the networks needed for pervasive computing and ubiquitous networking to be both, encompassing millions of elements in small areas many of whom exhibit fluttering availability and/or mobility. This is the inverse of what destination based routing achieves on the Internet. Volatility causes information to become stale rapidly, with staleness increasing with distance from origination. Volatility and scale conspire to increase the intractability of maintaining the routing information. Maintaining millions of routing entries in a low powered low cost device is not viable.

We are experimenting with novel and existing proposals to manage routing and forwarding information in large-scale volatile network environments. Some existing proposals employ source based routing where the sender specifies every node on the path to the intended destination. Intermediate nodes process the source route header to extract information about hosts on the path, creating a routing table of destinations. In addition to scalability problems of maintaining routing paths to each destination, source based routing fails to exploit the improvement in information closer to the destination. Indeed, this is one of the attractive attributes of destination based routing on the Internet, where routing is based on coarse grained (aggregate) information far from destinations, but becomes gradually refined at routers closer to the destination.

To achieve aggregation and avoid the need for detailed information to be maintained throughout the network we are experimenting with a combination of featherweight flows and virtual topologies (overlays). A particular problem as the number of network elements grows is that without route aggregation, the number of routes maintained at each node is likely to far exceed the number of flows active at the node at any given time. To combat this problem we break large networks into smaller regions, maintaining per-host information for nearby hosts but keeping track of regions rather than systems and paths in more distant parts of the network. While in many ways similar to self-configured IGP areas, the regions are constantly changing and may be overlapping. Featherweight flows, allow a fast creation of a tunnels/connections between regions and systems. A lightweight connection setup may be source routed or may carry a "report-route" type option to propagate information about hosts and connectivity. This way a tradeoff based on scale and volatility allows individual nodes to dynamically select a point in the spectrum from fully host based routing information to more aggregate virtual topology style routes. 


\subsection{Dependability of Connectivity and Network Services}

The second element of our effort is dependability of network connectivity. In wired networks where physical failures in connectivity are relatively rare, dependability translates into configuration management, dynamic routing updates, fast restorations and hot-spot diffusion. The wireless ad-hoc networks that we are studying require self-configuration of both node-local facilities and of inter-nodal state maintenance, in particular routing protocols. While automatic configuration avoids the misconfigurations that threaten the reliability of current production networks, self-configuration of routing protocols at the scale of interest is inherently tricky.

In more volatile environments maintaining basic connectivity is an ongoing effort of significant complexity. Fast restoration is replaced with continuing local search for the currently best available channel. However, whereas local scanning and rapid soft hand-offs can maintain local connectivity, rapid local restoration in wired networks assumes very low likelihood of multiple failures. This assumption is not valid in adhoc wireless networks. To achieve dependability we have devised a scheme similar to the one used to achieve scalability in routing, where network elements are grouped into regions. Within a region, the network nodes monitors the network connectivity to neighboring nodes, constructing a profile of dependability for these nodes. Using these profiles nodes select the most dependable nodes to form a set of virtual access points for that region. When routes fail, fast restoration and hot-spot diffusion exploit these virtual access points as intermediaries until new information restores better routes to replace those lost.

\subsection{Nodal Architecture and Operating Systems Support}

The third focus of our effort is on nodal architecture and operating system support for ubiquitous and ad-hoc networking. Over the last few years we have researched and implemented architecture for an active and programmable network node, and experimented with it as a network server, high-performance router, and opticalnetworking controller, with applications such as media streaming, multicast, and adaptive congestion control. The architecture supports a range of dynamically programmable activity, ranging from pure control plane functionality, to limited access the datapath, to intercepting and processing every datagram. The flexibility of this platform makes it attractive as a basis for ad-hoc networked elements and ubiquitous networking. These attributes make our nodal architecture an ideal platform to realize and experiment with the spectrum of choices in solving the issues detailed below, while gaining understanding of the requirements that ubiquitous networking imposes on the operating systems of network elements.

Our initial assessment is that our platform and more generally active and programmable networking approaches seem effective and beneficial in ubiquitous networking and pervasive computing environments. Favorable bandwidth to processing ratio - relatively low bandwidth compared to processing power - affords additional per packet processing when required. Programmability provides adaptability to the elements, allowing in-field adaptation and software upgrades. Our architecture has proven effective either as a networked end-system or as a router; instantiated as the nodal OS for ubiquitous network element and sensory equipment it now provides both functions. 


\section{References}

[1] Gísli Hjálmtýsson, "The Pronto Platform - A Flexible Toolkit for Programming Networks using a Commodity Operating System," in the proceedings of OpenArch 2000, Tel Aviv, Israel, March 2000.

[2] Gísli Hjálmtýsson, Heimir Sverrisson, Björn Brynjúlfsson and Ólafur R. Helgason, "Dynamic packet processors - A new abstraction for router extensibility," in the proceedings of OpenArch 2003, San Francisco, April 2003.

[3] Gísli Hjálmtýsson, Björn Brynjúlfsson and Ólafur Ragnar Helgason, "SLIM - Selfconfiguring Lightweight Internet Multicast," RU Technical Report. In preparation to be submitted for publication.

[4] Gísli Hjálmtýsson, Björn Brynjúlfsson and Ólafur Ragnar Helgason, "Overcoming lasthop/first-hop problems in IP multicast," RU Technical Report. Submitted for publication.

[5] Gísli Hjálmtýsson, Jennifer Yates, Sid Chaudhuri and Albert Greenberg, "Smart Routers . Simple Optics: An Architecture for the Optical Internet," IEEE/OSA Journal of Lightwave Technology, December 2000.

[6] Gísli Hjálmtýsson, Jennifer Yates and Sid Chaudhuri, "Restoration Services for the Optical Internet," in the proceedings SPIE.s Terabit Optical Networking: Architecture, Control and Management Issues conference, Boston, MA, November 2000.

[7] Gísli Hjálmtýsson, Panagiotis Sebos, Graham Smith, and Jennifer Yates, "Simple IP Restoration for IP/GbE/10GbE optical networks," Postdeadline paper PD-36, OFC 2000, Baltimore, MD, March 2000.

[8] Nikos Anerousis and Gísli Hjálmtýsson, "Service Level Routing on the Internet," in the proceedings of Globecom'99, Rio de Janeiro, Brazil, December 1999. 